\title{
To see and remember: Visually specific information is retained in memory from previously attended objects in natural scenes
}

\author{
ANDREW HOLLINGWORTH, CARRICK C. WILLIAMS, and JOHN M. HENDERSON \\ Michigan State University, East Lansing, Michigan
}

\begin{abstract}
What is the nature of the representation formed during the viewing of natural scenes? We tested two competing hypotheses regarding the accumulation of visual information during scene viewing. The first holds that coherent visual representations disintegrate as soon as attention is withdrawn from an object and thus that the visual representation of a scene is exceedingly impoverished. The second holds that visual representations do not necessarily decay upon the withdrawal of attention, but instead can be accumulated in memory from previously attended regions. Target objects in line drawings of natural scenes were changed during a saccadic eye movement away from those objects. Three findings support the second hypothesis. First, changes to the visual form of target objects (token substitution) were successfully detected, as indicated by both explicit and implicit measures, even though the target object was not attended when the change occurred. Second, these detections were often delayed until well after the change. Third, changes to semantically inconsistent target objects were detected better than changes to semantically consistent objects.
\end{abstract}

A traditional assumption in vision science is that the visual system constructs a global sensory image of the external world, for example by integrating sensory information over multiple eye fixations (Davidson, Fox, \& Dick, 1973; Feldman, 1985; McConkie \& Rayner, 1976). ${ }^{1}$ Such a representation could be used to support cognitive and behavioral competencies that rely on vision and would account for the phenomenological perception of a highly detailed and stable visual world. A large body of research, however, has demonstrated that the visual system does not construct this type of representation, since sensory information does not appear to be integrated across eye movements (Henderson, 1997; Irwin, 1991; Irwin, Yantis, \& Jonides, 1983; O'Regan \& Lévy-Schoen, 1983; Pollatsek \& Rayner, 1992; Rayner \& Pollatsek, 1983). More recently, research using naturalistic scene stimuli has arrived at a similar conclusion. Relatively large changes to a natural scene can go undetected if the change occurs during a saccadic eye movement or other visual disruption (Grimes, 1996; Henderson \& Hollingworth, 1999b; Henderson, Hollingworth, \& Subramanian, 1999; McConkie \& Currie, 1996; Rensink, O'Regan, \& Clark, 1997), an effect that has been termed change blindness (Rensink et al., 1997; for reviews see Hender-

This research was supported by a National Science Foundation (NSF) graduate fellowship to A. Hollingworth and by NSF Grants SBR 9617274 and ECS 9873531 to J. Henderson. We thank Laura Carlson, Keith Rayner, Ron Rensink, Dan Simons, and Tom Carr for helpful comments on a previous version of this manuscript and Gary Schrock for technical assistance. Correspondence should be addressed to A. Hollingworth, who is now at Yale University, Department of Psychology, Box 208205, New Haven, CT 06520-8205(e-mail: andrew.hollingworth@yale.edu). son \& Hollingworth, 1999a; Simons \& Levin, 1997). The logic behind these studies is that if a global sensory image is constructed, changes to the scene should be detected easily.

Recent proposals have abandoned the idea of a global sensory image in favor of the view that the visual representation of a scene is both local and transient, limited almost exclusively to the currently attended object (O'Regan, 1992; O’Regan, Rensink, \& Clark, 1999; Rensink, 2000a, 2000b; Rensink et al., 1997; Simons \& Levin, 1997; Wolfe, 1999). In this view, when attention is withdrawn from an object, the visual representation of that object decays immediately; consequently, the scene representation constructed during viewing and retained across disruptions such as eye movements is proposed to be exceedingly impoverished. Rensink (2000a, 2000b; Rensink et al., 1997) has provided the most detailed account of this view, which he has termed coherence theory, and which rests on four claims. First, attention is necessary to bind visual features into a coherent object representation and to preserve this representation in visual short-term memory (VSTM). Second, attended information in VSTM is stable across brief disruptions such as saccadic eye movements, supporting change detection, whereas unattended sensory representations decay rapidly and are overwritten by new visual input. Third, when visual attention is withdrawn from an object, the representation of that object immediately reverts to its preattentive state, becoming "unglued" (see also Wolfe, 1999). Fourth, initial perceptual processing of a scene activates representations of scene gist and general spatial layout, which are preserved across visual interruptions, providing an impression of scene continuity. 
Critically, because in coherence theory visual representations of local objects do not persist after the withdrawal of attention, the visual system is unable to accumulate information from previously attended regions. Coherence theory is therefore consistent with a view of perception in which the visual system does not rely heavily (or at all) on memory, but instead depends on the fact that local objects in the environment can be sampled when necessary by movements of the eyes or attention (Ballard, Hayhoe, Pook, \& Rao, 1997; O'Regan, 1992; O'Regan et al., 1999). The world itself serves as an "external memory."

A related proposal by Irwin (1992; Irwin \& Andrews, 1996) holds that the visual representation of an object does not necessarily disintegrate immediately upon the withdrawal of attention, but can be retained briefly in VSTM as part of an object file (see Kahneman, Treisman, \& Gibbs, 1992). However, due to the strong capacity limitations on VSTM, object files from previously attended objects will be quickly replaced as the eyes and attention are directed to other objects in a scene, and thus there will be little or no accumulation of visual information from previously attended regions. Therefore, as in coherence theory, the visual representation of local objects in a scene is proposed to be transient.

An alternative to these proposals holds that although the visual system does not construct a global sensory image, a relatively detailed scene representation can be formed nonetheless, retaining higher level visual representations in memory from previously attended objects. This visual memory theory rests on the following claims, discussed in detail in Hollingworth and Henderson (2002; see also Henderson \& Hollingworth, in press). First, when visual attention is directed to a scene region, in addition to sensory processing, higher level visual representations are formed that are abstracted away from sensory properties. Higher level visual representations can code quite detailed information regarding the visual form of an object, specific to the viewpoint at which the object was observed (Bülthoff, Edelman, \& Tarr, 1995; Riesenhuber \& Poggio, 1999), and representations specific to the visual form and orientation of an object can be retained across eye movements (Carlson-Radvansky, 1999; Carlson-Radvansky \& Irwin, 1995; Henderson \& Siefert, 1999, 2001; Pollatsek, Rayner, \& Collins, 1984). Second, as a consequence of being attended, the higher level visual representation of an object is consolidated into a more stable long-term memory representation. Thus, over multiple fixations on a scene, visual information from local objects accumulates in memory (along with conceptual information derived from object identification), forming a larger scale representation of that scene. This view is consistent with evidence that long-term memory for visual scenes is remarkably accurate. Participants are able to successfully recognize thousands of pictures presented earlier at study (Nickerson, 1965; Shepard, 1967; Standing, 1973; Standing, Conezio, $\&$ Haber, 1970). In addition, long-term scene memory does not appear to be limited to the gist of the scene. First, participants can accurately discriminate between studied scenes and mirror-reversed versions of the same scenes, which should not differ in their gist (Standing et al., 1970). Second, participants can successfully discriminate between individual objects that appeared in a studied scene and conceptually similar but visually different distractors, such as a different object from the same basic-level category or the same object in a different orientation (Friedman, 1979; Hollingworth \& Henderson, 2002; Parker, 1978).

The present experiment sought to test whether visual object representations disintegrate immediately upon the withdrawal of attention, as proposed by coherence theory, or whether such representations accumulate to form a relatively detailed representation of a scene, as proposed by visual memory theory. The experiment was not designed to test Irwin's view directly, though some of the results will be discussed in light of that proposal. In the present experiment, we examined participants' ability to detect changes to the visual form of individual objects appearing within line drawings of natural scenes. During free viewing of a scene, a target object was changed by replacing that object with a visually different object from the same basic-level category, as illustrated in Figure 1 . These token changes were made during a saccadic eye movement that took the eyes away from the target object after the eyes had fixated it for the first time. Visual attention and eye position are linked during normal viewing, with attention automatically preceding the eyes to the next saccade target (Deubel \& Schneider, 1996; Henderson, Pollatsek, \& Rayner, 1989; Hoffman \& Subramanian, 1995; Kowler, Anderson, Dosher, \& Blaser, 1995; Rayner, McConkie, \& Ehrlich, 1978; Shepherd, Findlay, \& Hockey, 1986). Thus, the target object was not within the current focus of visual attention when it changed: Before the initiation of the eye movement that triggered the change, visual attention had shifted from the target object to the next saccade target.

Coherence theory holds that only changes to currently attended objects can be detected, because representations preserving information specific to object tokens are maintained only in the presence of attention (Rensink, 2000a). More generally, coherence theory predicts that if a scene change occurs during a saccadic eye movement or other visual disruption, that change should be detected only if it alters attended visual information, the layout, or the gist of the scene. Thus, this hypothesis predicts that a token change made during a saccade away from the target object should not be detected, since the change does not alter attended visual information, the layout, or the gist of the scene. In contrast, visual memory theory holds that the representation of a previously attended object can be sufficiently detailed to support the detection of changes to the visual form of that object.

We also included a manipulation to provide an additional test of visual memory theory. The target object was either semantically consistent with the scene (e.g., a 

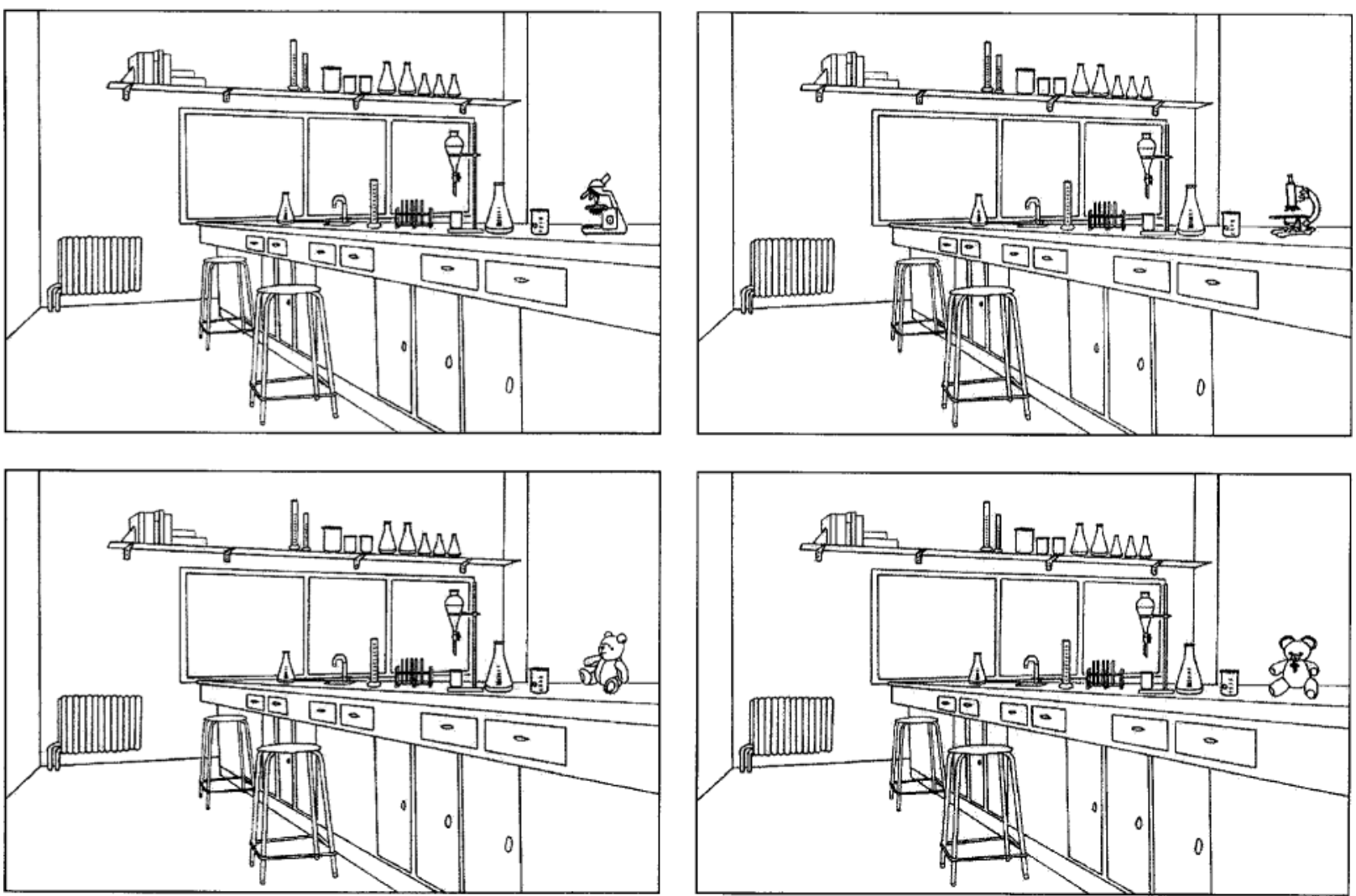

Figure 1. An example of a stimulus scene. The top panels illustrate a token change for a semantically consistent target object (microscope), and the bottom panels illustrate a token change for a semantically inconsistent object (teddy bear). The laboratory scene was paired with a bedroom scene in which the teddy bears were consistent and the microscopes inconsistent.

microscope in a laboratory) or semantically inconsistent (e.g., a teddy bear in a laboratory), as illustrated in Figure 1. Prior research has demonstrated that semantically inconsistent objects are retained more accurately in long-term memory than are semantically consistent objects (Friedman, 1979; Pedzek, Whetstone, Reynolds, Askari, \& Dougherty, 1989). If the scene representation constructed during extended viewing retains detailed visual information in memory from previously attended objects, then performance in this experiment would be expected to reflect the established characteristics of scene memory. Thus, we expected to find a change detection advantage for semantically inconsistent objects. In addition, such an effect would replicate the finding that changes to semantically inconsistent objects are detected sooner and more accurately than are changes to consistent objects (Hollingworth \& Henderson, 2000).

\section{METHOD}

Twelve naive participants viewed 24 black-on-white line drawings of natural scenes presented on a computer monitor for $20 \mathrm{sec}$ each. Fourteen scenes were generated from those used by van Diepen and De Graef (1994), and the other 10 scenes were generated from photographs taken in the East Lansing, Michigan area. The images generated from the two sources were not distinguishable. The main contours of the scenes were traced using commercial software to create line drawings. For each scene, two semantically consistent target objects (different tokens of the same basic-level category) were created by digitally tracing scanned images. Target objects were then switched between scenes to create the semantically inconsistent condition. This paired-scene design assured that the visual magnitude of the changes was controlled across consistency conditions. All target objects appeared in the same position in each scene, which did not coincide with the experimenter-determined initial fixation position. This position was chosen as a place within the scene where the consistent target objects might reasonably appear. Scene images subtended $15.8^{\circ}$ by $11.9^{\circ}$ of visual angle at a viewing distance of $1.13 \mathrm{~m}$. Target objects subtended an average of $1.64^{\circ}$ along the longest axis.

Eye movements were monitored using a Generation 5.5 Fourward Technologies dual Purkinje-image eyetracker that provided $1^{\prime}$ arc accuracy. Eye position was sampled at a rate better than $1000 \mathrm{~Hz}$. A critical region was defined around the target object in each scene, extending $0.36^{\circ}$ on each side beyond the smallest rectangle that could enclose that object. Token changes were triggered when the eyes crossed the critical region boundary on the saccade away from the target object after it had been fixated the first time. To ensure that the target object had been fixated prior to the change, the critical region was not activated until the eyes had dwelled within the target object region for a continuous period of at least $25 \mathrm{msec}$. Vertical refresh was set at $143 \mathrm{~Hz}$, and object changes were completed in less than $14 \mathrm{msec}$.

Participants were instructed to view each scene to prepare for a memory test on which a small detail of a single object might be dif- 
ferent (preparation for a memory test was a cover story designed to ensure that participants would examine the entire scene; no memory test was administered). Participants were also instructed to monitor for object changes during initial viewing and to press a button as soon as a change was detected. The token change manipulation was demonstrated using a sample scene. These instructions were the same as those used in Henderson and Hollingworth (1999b) and similar to the instructions used in other studies demonstrating transsaccadic change blindness (e.g., Grimes, 1996). Each participant saw all 24 scene items. On 16 trials, the target object was changed. Eight control trials were included on which the target object was not changed. Within each of these conditions, half of the scenes contained a semantically consistent target object and the other half a semantically inconsistent target object. Across participants, each scene appeared in each condition an equal number of times. The order of scene presentation was determined randomly for each participant.

\section{RESULTS AND DISCUSSION}

Trials were eliminated if the eyetracker lost track of eye position prior to the change or if the change was not completed before the beginning of the next fixation on the scene. These trials accounted for $12.0 \%$ of the data. The principal data discussed below are presented in Table 1.

\section{Explicit Measure of Detection}

We first examined the percentage of trials on which the participant pressed the response button to indicate that a change had been detected. Overall, $26.6 \%$ of changes were detected. This detection rate was reliably different from the $2.1 \%$ false alarm rate in the control condition $\left[F(1,11)=37.29, M S_{\mathrm{e}}=194.1, p<.001\right]$. Supporting the prediction derived from visual memory theory, participants could sometimes detect token changes even though the object was not focally attended when the change occurred. Although change detection performance was nowhere near perfect, coherence theory holds that detection of token changes should never occur in the absence of attention and therefore cannot account for even this modest level of performance. Thus, these results demonstrate that participants are not always blind to changes that occur outside of the current focus of attention.

Further support for visual memory theory derives from the fact that change detection was often delayed until well after the change occurred. A significant percentage (41\%) of button presses were registered more than $1,500 \mathrm{msec}$ after the change. ${ }^{2}$ Of these "late detections," $94 \%$ oc- curred when the target object was refixated later in viewing. This finding suggests that detailed visual information was often retained for a relatively long period of time and consulted only when focal attention was directed back to the changed region. Such evidence of delayed detection is not consistent with coherence theory, and it is also difficult to explain under Irwin's theory. The latter view holds that although a visual object representation can be retained in VSTM after the withdrawal of attention from an object, that representation should be replaced very quickly as attention is directed to other objects in the scene.

Converging evidence in support of visual memory theory comes from Henderson and Hollingworth (1999b) and Holling worth and Henderson (2002). In these studies, we found that participants could detect a different type of visual change $\left(90^{\circ}\right.$ in-depth rotation of the target object) on a significant percentage of trials when the change occurred during a saccade away from that object or during a saccade to a different object in the scene. In neither of these cases was the target object attended when the change occurred. As in the present experiment, change detection was often delayed until refixation of the changed object. These data also eliminate a potential alternative explanation for performance in the present experiment. It is possible that detection of some token changes could be mediated by conceptual representations at a subordinate level of categorization (e.g., a hand drill was replaced by a power drill in the workshop scene). However, successful detection of orientation changes after the withdrawal of attention provides strong converging evidence that the representations supporting change detection were visual, since a rotation change does not alter the conceptual identity of the target object at all.

In addition to predicting above-floor change detection performance, visual memory theory predicted an advantage for the detection of changes to semantically inconsistent objects. This pattern was observed, with better detection of changes to inconsistent targets $(35.2 \%)$ than to consistent targets $(18.1 \%)\left[F(1,11)=5.28, M S_{\mathrm{e}}=\right.$ $334.0, p<.05]$. The amplitude of the saccadic eye movement that triggered the change did not differ as a function of semantic consistency. For change trials, the mean amplitude was $4.03^{\circ}$ in the consistent condition and $4.20^{\circ}$ in the inconsistent condition $(F<1)$. Thus, differences in detection performance as a function of consis-

Table 1

Measures of Change Detection and Eye Movement Behavior

\begin{tabular}{|c|c|c|c|c|}
\hline \multirow[b]{2}{*}{ Measure } & \multicolumn{2}{|c|}{ Token Change } & \multicolumn{2}{|c|}{ Control } \\
\hline & Consistent & Inconsistent & Consistent & Inconsistent \\
\hline$\%$ correction detection & 18.1 & 35.2 & - & - \\
\hline$\%$ false alarms & - & - & 4.2 & 0.0 \\
\hline $\begin{array}{l}\text { Gaze duration on target } \\
\text { for first entry after } \\
\text { change (in milliseconds) }\end{array}$ & $753^{*}$ & $744 *$ & 419 & 579 \\
\hline
\end{tabular}


tency cannot be explained by differences in the quality of the visual information available directly after the change.

This semantic consistency effect was likely due, at least in part, to the fact that inconsistent targets were fixated longer than consistent targets prior to the change. Mean gaze duration was $628 \mathrm{msec}$ in the former condition and $489 \mathrm{msec}$ in the latter $\left[F(1,11)=7.46, M S_{\mathrm{e}}=\right.$ $15,751, p<.05$ ] (gaze duration is the sum of all fixation durations from the point at which the eyes enter an object region to the point when they leave that region). These data suggest that differences in initial encoding time could be responsible for the effect of semantic consistency on change detection rather than differences in object memory per se. To investigate this issue, we examined more closely the relationship between detection performance and gaze duration on the target. Figure 2 displays detection performance in each consistency condition as a function of gaze duration on the target prior to the change. Change detection was consistently higher for inconsistent targets throughout the gaze duration range, suggesting that there was an effect of semantic consistency above that attributable to differences in initial encoding time (see Friedman, 1979, for similar results in a long-term memory paradigm). Thus, the results of the present experiment replicate the inconsistent object advantage found within the scene memory and change detection literatures, and provide converging evidence for a memory locus of change detection effects in this study.

In summary, the explicit detection data support the conclusion that the online representation of a scene can contain detailed visual information in memory from previously attended objects. Contrary to the proposal of coherence theory, the results indicate that visual object rep- resentations do not disintegrate upon the withdrawal of attention. In addition, when a scene change is made during a saccadic eye movement, the current data demonstrate that the continuous allocation of visual attention to the changing region at the time of change is not a necessary condition for change detection.

\section{Implicit Measure of Detection}

We also examined the possibility that there might be effects of change not captured by the explicit detection measure. For trials without explicit detection, we calculated the length of time the eyes fixated the target object after the change as a function of whether the object was changed or not (in the control condition, a "change" consisted of replacing the initial scene image with an identical image). Gaze duration on the target object was examined for the first entry of the eyes into that region after the change, both for misses in the token-change condition and for the equivalent entry in the control condition. There was a main effect of change, with reliably longer mean gaze duration in the token-change condition (749 msec) than in the control condition $(499 \mathrm{msec})$ $\left[F(1,11)=6.29, M S_{\mathrm{e}}=118,933, p<.05\right]$. The effect of semantic consistency was not reliable $(F<1)$, and neither was the interaction $(F<1.25)$. This implicit effect of change on gaze duration replicates a similar effect in a study using 3-D rendered color images of scenes (Hollingworth \& Henderson, 2002).

As with the explicit detection results, the implicit effect was often obtained well after the change occurred. For misses, the mean elapsed time between the change and the first reentry of the eyes into the target region was $5.16 \mathrm{sec}$ and did not differ as a function of semantic consistency $(F<1)$. It is exceedingly unlikely that the target

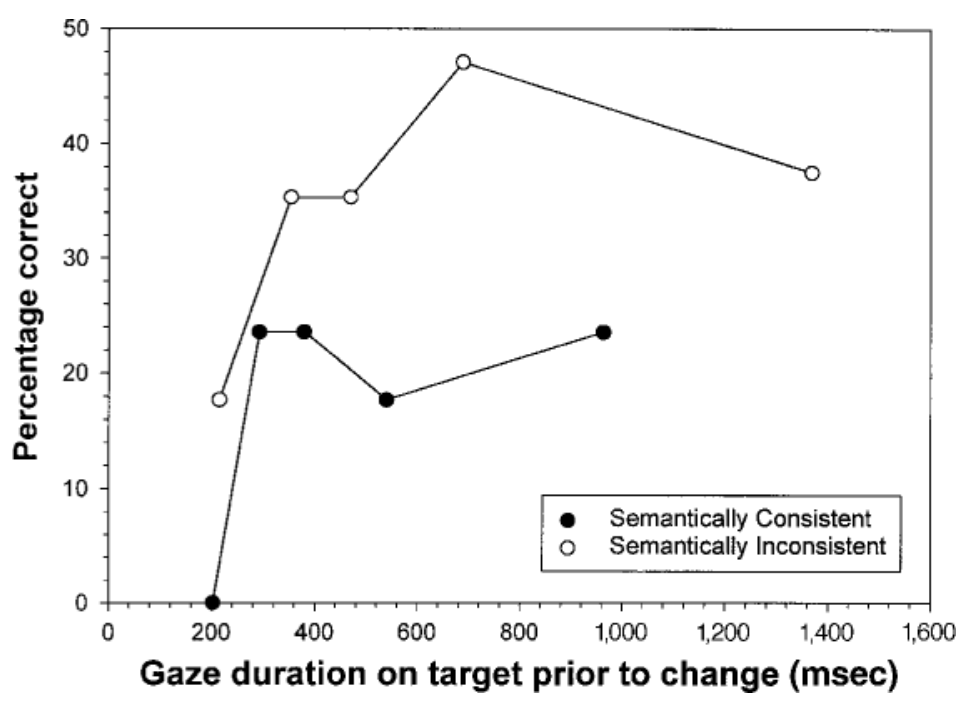

Figure 2. Mean percentage correct change detection performance as a function of sem antic consistency and gaze duration on the target object prior to the change. For the two consistency conditions, the mean of each gaze duration quintile is plotted against mean change detection performance in that quintile. 
object was attended continuously during this period, because the eyes were actively fixating other objects in the scene (there was an average of 13.5 fixations between the token change and the first reentry). Thus, these data provide further evidence that visual object representations do not decay upon the withdrawal of attention: Visually specific information was retained in memory across multiple intervening eye fixations and influenced subsequent fixation time on the changed object.

What do implicit effects reveal about the underlying representation of objects in scenes? Prior reports have raised the possibility that explicit and implicit change detection effects reflect the operation of two different forms of visual representation: one that is available to awareness (supporting explicit effects) and another that is unavailable to awareness (supporting implicit effects) (Fernandez-Duque \& Thornton, 2000; Simons, 2000). It is important to note, however, that this need not be the case. Effects of change on an implicit measure such as gaze duration do not necessarily indicate that the information causing the effect was itself implicit (i.e., unavailable to awareness). Effects on gaze duration in this study could plausibly have resulted from the accumulation of the same type of information as supported explicit detection; however, on miss trials this accumulation was simply not sufficient to exceed a threshold for explicit response (see also Williams \& Simons, 2000). In any case, effects of change on implicit measures clearly demonstrate that the representation of a scene is significantly more detailed than explicit detection results imply.

\section{Change Blindness Reconsidered}

Given that visual object representations appear to be reliably retained in memory after attention has been withdrawn from an object, it is important to examine why other studies have found exceedingly poor change detection, leading to the conclusion that the internal representation of a scene is impoverished. First, in studies demonstrating change blindness, eye movements have rarely been monitored, and thus the critical change in the scene may occur before the target region is fixated. A number of studies have demonstrated that the encoding of local scene information is strongly dependent on fixation position (Hollingworth \& Henderson, 2002; Hollingworth, Schrock, \& Henderson, 2001; Nelson \& Loftus, 1980). For example, Hollingworth and Henderson (2002) found that if a target object had not been fixated prior to changing that object, change detection performance was no higher than the false alarm rate. Given this dependence of encoding on fixation, change blindness may occur, in part, because target objects are not always fixated prior to a change and detailed information has not been encoded from that object. In addition, Hollingworth et al. (2001) monitored eye movements in a periodic change (or flicker) paradigm and found that the large majority of changes were detected when the target object was directly fixated (see also O'Regan, Deubel, Clark, \& Rensink, 2000). Thus, the finding that changes to more informative objects are detected more quickly than changes to less informative objects in the flicker paradigm (Rensink et al., 1997) is very likely due to the well-established fact that more informative objects tend to be fixated earlier, longer, and more often during scene viewing (Antes, 1974; Friedman, 1979; Henderson, Weeks, \& Hollingworth, 1999; Loftus \& Mackworth, 1978).

A second reason change blindness might occur despite the accumulation of relatively detailed object representations from previously attended regions of a scene is that the relevant object representation may not be reliably retrieved to support change detection. The present experiment (see also Henderson \& Holling worth, 1999b; Hollingworth \& Henderson, 2002) demonstrated that refixation plays a significant role in the retrieval of scene information needed to support change detection. Again, since most change detection studies have not monitored eye movements, changed regions may not be refixated on a significant number of trials. If the changed region is not refixated, then the change may go undetected despite the fact that the stored representation of that object is sufficiently detailed to support change detection.

Finally, the traditional interpretation of change detection performance may be incorrect. Researchers in the change blindness literature have tended to use the following logic. Explicit change detection directly reflects the extent to which scene information is represented. Therefore, if a change is not detected, the information necessary to detect the change must be absent from the internal representation of the scene. However, the results of this study demonstrating a large implicit effect of change on fixation duration, along with other recent demonstrations of implicit detection (Fernandez-Duque \& Thornton, 2000; Hayhoe, Bensinger, \& Ballard, 1998; Hollingworth \& Henderson, 2002; Williams \& Simons, 2000), reveal that explicit change detection provides a poor measure of the detail of the scene representation. Our results go further than prior reports by demonstrating that implicit effects can be obtained many seconds after the change and for an object that was not focally attended when the change occurred. Thus, change blindness may be observed not because the critical information is absent from the scene representation but because explicit change detection is not sensitive to the presence of that information.

\section{Conclusion}

The visual perception of natural scenes is a dynamic process during which local objects are selected by movements of the eyes and attention. The selective nature of scene perception, however, does not necessarily entail that visual representation is limited to the currently selected object. The results of this study demonstrate that visual object representations are retained in memory from previously attended regions of a scene. When attention is withdrawn from an object, there are indeed representational consequences of having attended that object. Thus, the present study supports a view of scene 
perception in which memory and perceptual processing are functionally integrated.

\section{REFERENCES}

ANTES, J. R. (1974). The time course of picture viewing. Journal of Experimental Psychology, 103, 62-70.

Ballard, D. H., Hay hoe, M. M., Pook, P. K., \& Rao, R P. (1997). Deictic codes for the embodiment of cognition. Behavioral \& Brain Sciences, 20, 723-767.

Biederman, I. (1987). Recognition-by-components: A theory of human image understanding. Psychological Review, 94, 115-147.

Bülthoff, H. H., EdElman, S. Y., \& TARR, M. J. (1995). How are threedimensional objects represented in the brain? Cerebral Cortex, $\mathbf{3}$, 247-260

Carlson-Radvansky, L. A. (1999). Memory for relational information across eye movements. Perception \& Psychophysics, 61, 919-934.

Carlson-Radvansky, L. A., \& IRwin, D. E. (1995). Memory for structural information across eye movements. Journal of Experimental Psychology: Learning, Memory, \& Cognition, 21, 1441-1458.

Davidson, M. L., Fox, M. J., \& Dick, A. O. (1973). Effect of eye movements on backward masking and perceived location. Perception \& Psychophysics, 14, 110-116.

Deubel, H., \& Schneider, W. X. (1996). Saccade target selection and object recognition: Evidence for a common attentional mechanism. Vision Research, 36, 1827-1837.

Feldman, J. A. (1985). Four frames suffice: A provisional model of vision and space. Behavioral \& Brain Sciences, 8, 265-289.

Fernandez-Duque, D., \& Thornton, I. M. (2000). Change detection without awareness: Do explicit reports underestimate the representation of change in the visual system? Visual Cognition: Special Issue on Change Detection \& Visual Memory, 7, 324-344.

Friedman, A. (1979). Framing pictures: The role of knowledge in automatized encoding and memory for gist. Journal of Experimental Psychology: General, 108, 316-355.

GRIMES, J. (1996). On the failure to detect changes in scenes across saccades. In K. Akins (Ed.), Perception: Vancouver studies in cognitive science (Vol. 5, pp. 89-110). Oxford: Oxford University Press.

Hayhoe, M. M., Bensinger, D. G., \& Ballard, D. H. (1998). Task constraints in visual working memory. Vision Research, 38, 125-137.

Henderson, J. M. (1997). Transsaccadic memory and integration during real-world object perception. Psychological Science, 8, 51-55.

Henderson, J. M., \& Hollingworth, A. (1999a). High-level scene perception. Annual Review of Psychology, 50, 243-271.

Henderson, J. M., \& Hollingworth, A. (1999b). The role of fixation position in detecting scene changes across saccades. Psychological Science, 10, 438-443.

Henderson, J. M., \& Hollingworth, A. (in press). Eye movements, visual memory, and scene representation. In M. A. Peterson \& G. Rhodes (Eds.), Analytic and holistic processes in the perception of faces, objects, and scenes. Norwood, NJ: JAI/Ablex.

Henderson, J. M., Hollingworth, A., \& Subramanian, A. N. (1999, November). The retention and integration of scene information across saccades: A global change blindness effect. Paper presented at the annual meeting of the Psychonomic Society, Los Angeles.

Henderson, J. M., Pollatsek, A., \& Rayner, K. (1989). Covert visual attention and extrafoveal information use during object identification. Perception \& Psychophysics, 45, 196-208.

Henderson, J. M., \& Siefert, A. B. (1999). The influence of enantiomorphic transformation on transsaccadic object integ ration. Journal of Experimental Psychology: Human Perception \& Performance, 25, 243-255.

Henderson, J. M., \& Siefert, A. B. C. (2001). Types and tokens in transsaccadic object integration. Psychonomic Bulletin \& Review, 8 , $753-760$

Henderson, J. M., Weeks, P. A., Jr., \& Hollingworth, A. (1999). The effects of semantic consistency on eye movements during complex scene viewing. Journal of Experimental Psychology: Human Perception \& Performance, 25, 210-228.

Hoffman, J. E., \& Subramanian, B. (1995). The role of visual attention in saccadic eye movements. Perception \& Psychophysics, 57, 787-795
Hollingworth, A., \& Henderson, J. M. (2000). Semantic informativeness mediates the detection of changes in natural scenes. Visual Cognition, 7, 213-235.

Hollingworth, A., \& Henderson, J. M. (2002). Accurate visual memory for previously attended objects in natural scenes. Manuscript submitted for publication.

Hollingworth, A., Schrock, G., \& Henderson, J. M. (2001). Change detection in the flicker paradigm: The role of fixation position within the scene. Memory \& Cognition, 29, 296-304.

IRWIN, D. E. (1991). Information integration across saccadic eye movements. Cognitive Psychology, 23, 420-456.

IRWIN, D. E. (1992). Visual memory within and across fixations. In K. Rayner (Ed.), Eye movements and visual cognition: Scene perception and reading (pp. 146-165). New York: Springer-Verlag.

IRWIN, D. E., \& ANDREWS, R. (1996). Integration and accumulation of information across saccadic eye movements. In T. Inui \& J. L. McClelland (Eds.), Attention and performance XVI: Information integration in perception and communication (pp. 125-155). Cambridge, MA: MIT Press, Bradford Books.

Irwin, D. E., Yantis, S., Jonides, J. (1983). Evidence against visual integration across saccadic eye movements. Perception \& Psychophysics, 34, 49-57.

Kahneman, D., Treisman, A., \& GibBs, B. J. (1992). The reviewing of object files: Object-specific integration of information. Cognitive Psychology, 24, 175-219.

Kowler, E., Anderson, E., Dosher, B., \& Blaser, E. (1995). The role of attention in the programming of saccades. Vision Research, 35, 1897-1916.

Loftus, G. R., \& Mackworth, N. H. (1978). Cognitive determinants of fixation location during picture viewing. Journal of Experimental Psychology: Human Perception \& Performance, 4, 565-572.

MARr, D. (1982). Vision. San Francisco: W. H. Freeman.

McConkie, G. W., \& Currie, C. B. (1996). Visual stability while viewing complex pictures. Journal of Experimental Psychology: Human Perception \& Performance, 22, 563-581.

McConkie, G. W., \& RAYner, K. (1976). Identifying the span of the effective stimulus in reading: Literature review and theories of reading. In H. Singer \& R. B. Ruddell (Eds.), Theoretical models and processes in reading (pp.137-162). Newark, DE: International Reading Association.

Nelson, W. W., \& Loftus, G. R. (1980). The functional visual field during picture viewing. Journal of Experimental Psychology: Human Learning \& Memory, 6, 391-399.

NiCKERSON, R. S. (1965). Short-term memory for complex meaningful visual configurations: A demonstration of capacity. Canadian Journal of Psychology, 19, 155-160.

O'Regan, J. K. (1992). Solving the "real" mysteries of visual perception: The world as an outside memory. Canadian Journal of Psychology, 46, 461-488.

O'Regan, J. K., Deubel, H., Clark, J. J., \& Rensink, R. A. (2000). Picture changes during blinks: Looking without seeing and seeing without looking. Visual Cognition, 7, 191-211.

O'Regan, J. K., \& LÉvy-Schoen, A. (1983). Integrating visual information from successive fixations: Does trans-saccadic fusion exist? Vision Research, 23, 765-768.

O'Regan, J. K., Rensink, R. A., \& Clark, J. J. (1999). Change blindness as a result of "mudsplashes." Nature, 398, 34.

Palmer, S. E. (1977). Hierarchical structure in perceptual representation. Cognitive Psychology, 9, 441-474.

PARKER, R. E. (1978). Picture processing during recognition. Journal of Experimental Psychology: Human Perception \& Performance, $\mathbf{4}$, 284-293.

Pedzek, K., Whetstone, T., Reynolds K., Askari, N., \& Dougherty, T. (1989). Memory for real-world scenes: The role of consistency with schema expectations. Journal of Experimental Psychology: Learning, Memory, \& Cognition, 15, 587-595.

PollatseK, A., \& RAyner, K. (1992). What is integrated across fixations? In K. Rayner (Ed.), Eye movements and visual cognition: Scene perception and reading (pp. 166-191). New York: Springer-Verlag.

Pollatsek, A., Rayner, K., \& Collins, W. E. (1984). Integrating pictorial information across eye movements. Journal of Experimental Psychology: General, 113, 426-442. 
Rayner, K., McConkie, G. W., \& Ehrlich, S. (1978). Eye movements and integrating information across fixations. Journal of Experimental Psychology: Human Perception \& Performance, 4, 529-544.

Rayner, K., \& Pollatsek, A. (1983). Is visual information integrated across saccades? Perception \& Psychophysics, 34, 39-48.

Rensink, R. A. (2000a). The dynamic representation of scenes. Visual Cognition: Special Issue on Change Detection \& Visual Memory, 7, 17-42.

Rensink, R. A. (2000b). Seeing, sensing, and scrutinizing. Vision Research, 40, 1469-1487.

Rensink, R. A., O' Regan, J. K., \& Clark, J. J. (1997). To see or not to see: The need for attention to perceive changes in scenes. Psychological Science, 8, 368-373.

Riesenhuber, M., \& Poggio, T. (1999). Hierarchical models of object recognition in cortex. Nature Neuroscience, 2, 1019-1025.

ShePARD, R. N. (1967). Recognition memory for words, sentences, and pictures. Journal of Verbal Learning \& Verbal Behavior, 6, 156-163.

Shepherd, M., Findlay, J. M., \& Hockey, R. J. (1986). The relationship between eye movements and spatial attention. Quarterly Journal of Experimental Psychology, 38A, 475-491.

Simons, D. J. (2000). Current approaches to change blindness. Visual Cognition: Special Issue on Change Detection \& Visual Memory, 7, $1-16$.

Simons, D. J., \& Levin, D. T. (1997). Change blindness. Trends in Cognitive Sciences, 1, 261-267.

SperLING, G. (1960). The information available in brief visual presentations. Psychological Monographs, 74 (11, Whole No. 498).

STANDING, L. (1973). Learning 10,000 pictures. Quarterly Journal of Experimental Psychology, 25, 207-222.

Standing, L., Conezio, J., \& Haber, R. N. (1970). Perception and memory for pictures: Single-trial learning of 2500 visual stimuli. Psychonomic Science, 19, 73-74. van Diepen, P. M. J., \& De Graef, P. (1994). Line-drawing library and software toolbox. (Psych. Rep. No. 165). Leuven, Belgium: University of Leuven, Laboratory of Experimental Psychology.

Williams, P., \& Simons, D. J. (2000). Detecting changes in novel 3D objects: Effects of change magnitude, spatiotemporal continuity, and stimulus familiarity. Visual Cognition: Special Issue on Change Detection \& Visual Memory, 7, 297-322.

Wolfe, J. M. (1999). Inattentional Amnesia. In V. Coltheart (Ed.), Fleeting memories (pp. 71-94). Cambridge: MIT Press.

\section{NOTES}

1. We take sensory information or sensory representation to mean a precategorical, maskable, complete, precise, and metrically organized "iconic" representation of the properties available from early vision (such as shape, shading, texture, color, etc.) (Irwin 1992; Sperling, 1960). The visual system also produces higher level representations abstracted away from sensory information. Candidate representations include structural descriptions (e.g., Biederman, 1987; Marr, 1982; Palmer, 1977) or other hierarchical representations of object form (e.g., Riesenhuber \& Poggio, 1999). We use the term visual to refer to both low-level sensory representations and higher level visual representations. In addition, we distinguish visual representations (encoding properties such as shape and color) from conceptual representations (encoding object identity and other associative information).

2. In a previous study (Henderson \& Hollingworth, 1999b), if a detection happened more than $1,500 \mathrm{msec}$ after the change, that detection almost always occurred upon refixation of the target object.

(Manuscript received August 22, 2000; revision accepted for publication January 24, 2001.)

\section{Call for Papers \\ BRMIC Special Issue on Eye Movement Research Methods}

Under the joint auspices of ACM ETRA (Association for Computing Machinery's Eye Tracking Research and Applications, see http://www.vr.clemson.edu/eyetracking/etra/2002) and Behavior Research Methods, Instruments, \& Computers, the November 2002 issue of BRMIC will feature articles that address research methods and instrumentation related to the study of oculomotor behavior. Papers are welcomed from a variety of areas (such as Computer Science and Human-Computer Interaction; Cognitive and Social Psychology; Ergonomics; Cognitive Neuroscience; and Applied Psychology).

All interested authors are invited to submit articles for review, via a PDF file attachment to an e-mail to brmic@ @amilton.edu., prepared according to the Information for Contributors in Volume 33 (3) of BRMIC or at http://www.psychonomic.org/BRMIC/manuscript.htm

Contributions should be conspicuously marked "For the Eye Tracking issue" and be sent to the editorial office no later than March 15, 2002. Questions about submission content and format may be addressed to the General Chair of ETRA, Andrew T. Duchowski (andrewd@cs.clemson.edu) or to the editor of BRMIC, Jonathan Vaughan (brmic@ hamilton.edu). 